\title{
ON THE NON-EXISTENCE OF CERTAIN CLASSES OF GENERALIZED BENT FUNCTIONS
}

\author{
CHANG LV AND JIANING LI
}

\begin{abstract}
We obtain new non-existence results of generalized bent functions from $\mathbb{Z}_{q}^{n}$ to $\mathbb{Z}_{q}$ (called type $[n, q]$ ). The first case is a class of types where $q=2 p_{1}^{r_{1}} p_{2}^{r_{2}}$. The second case contains two types $\left[1 \leq n \leq 3,2 \times 31^{e}\right]$ and $\left[1 \leq n \leq 7,2 \times 151^{e}\right]$.
\end{abstract}

\section{INTRODUCTION}

Let $q \geq 2$ be an integer, $\mathbb{Z}_{q}=\mathbb{Z} / q \mathbb{Z}, \zeta_{q}=\exp \left(\frac{2 \pi i}{q}\right)$.

Definition 1.1. A function $f$ from $\mathbb{Z}_{q}^{n}$ to $\mathbb{Z}_{q}$ is called a Generalized Bent Function $(G B F)$ of type $[n, q]$ if

$$
F(\lambda) \overline{F(\lambda)}=q^{n}
$$

for every $\lambda \in \mathbb{Z}_{q}^{n}$ where

$$
F(\lambda)=\sum_{x \in \mathbb{Z}_{q}^{n}} \zeta_{q}^{f(x)} \cdot \zeta_{q}^{-x \cdot \lambda}
$$

is the Fourier transform of the function $\zeta_{q}^{f(x)}, x \cdot \lambda$ is the standard dot product, and $\overline{F(\lambda)}$ is the complex conjugate of $F(\lambda)$.

Bent functions were first introduced by Rothaus [16 in 1976, and were generalized to GBFs by Kumar et al. 9] in 1985. GBFs have been used in many fields such as difference sets, coding theory, cryptography and sequence designs. For more background information and its applications we refer the reader to [2, 14, 9].

A natural question is when bent functions do exist. Rothaus [16 proved that bent functions from $\mathbb{Z}_{2}^{n}$ to $\mathbb{Z}_{2}$ exist if and only if $n$ is even. For GBFs defined in [1.1, Kumar et al. 9] constructed them except the case that $n$ is odd and $q \equiv 2(\bmod 4)$.

From now on we assume $n$ is odd and $q=2 N$ with $2 \nmid N \geq 3$ So far there is no GBF being constructed in this case, while there are many non-existence results under some extra constraints. We give a list of these results with reference at the beginning of each item:

(1) $($ Kummar 9$]) 2^{s} \equiv-1(\bmod N)$ for some integer $s \geq 1$,

(2) (Ikeda [7]) type $\left[1,2 p_{1}^{e_{1}} \ldots p_{g}^{e^{g}}\right]$ where $p_{1}, \ldots, p_{g}$ are distinct primes and $p_{i}^{s_{i}} \equiv-1\left(\bmod N / p_{i}^{e_{i}}\right)$ for some $s_{i}, i=1, \ldots, g$.

Date: September 22, 2018.

2000 Mathematics Subject Classification. 11R04, 94A15, 13C20.

Key words and phrases. Generalized bent functions, Cyclotomic fields, Class groups, Stickelberger relations. 
By (1.2) in the definition, if there is no element in $\mathbb{Z}\left[\zeta_{q}\right]$ with absolute value $q^{\frac{n}{2}}$, i.e.

$$
\alpha \bar{\alpha}=q^{n},
$$

has no solution in $\mathbb{Z}\left[\zeta_{q}\right]$. then there is no GBF of type $[n, q]$. Using this fact, Feng et al. [4, 5, 3] obtain the following non-existence results:

(3) (Feng [4]) type $\left[n<m / s, 2 p^{l}\right]$, where $p \equiv 7(\bmod 8)$ is a prime, $s=\frac{\varphi\left(p^{l}\right)}{\operatorname{ord}_{p l}(2)}$ and $m$ is the smallest odd positive integer s.t. $x^{2}+p y^{2}=2^{m+2}$ has integral solutions,

(4) (Feng et al. [4, 5, 3, 11]) various classes of type $\left[n<m, 2 p_{1}^{l_{1}} p_{2}^{l_{2}}\right]$, where $p_{1}, p_{2}$ are two distinct primes satisfy some conditions and $m$ is an upper bound for $n$.

However, in the case that (1.3) is solvable over $\mathbb{Z}\left[\zeta_{q}\right]$, one has to use other methods to obtain non-existence results, which are typically collected in the following:

(5) (Pei [15]) type $[1,2 \times 7]$,

(6) (Ikeda 7]) see (2) before,

(7) (Jiang and Deng [8] $)\left[3,2 \times 23^{e}\right]$,

(8) (Li and Deng [10]) type $\left[m, 2 p^{e}\right]$ where $p \equiv 7(\bmod 8)$ is a prime with $\operatorname{ord}_{p^{e}}(2)=\varphi\left(p^{e}\right) / 2$ and $m$ is defined the same as in (3).

In this article we extend the results where $q=2 p^{e}$ in (8) to the case where $q=p_{1}^{r_{1}} p_{2}^{r_{2}}$ :

Theorem 1.4. Let $N=p_{1}^{r_{1}} p_{2}^{r_{2}}$ where $r_{1}, r_{2} \geq 1$. Let $p_{1} \equiv 7(\bmod 8)$ and $p_{2} \equiv 5(\bmod 8)$ be two primes satisfy

(i) $\operatorname{ord}_{N}(2)=\varphi(N) / 2$, where $\varphi$ is the Euler phi function;

(ii) there is a positive integer s such that $p_{1}^{s} \equiv-1\left(\bmod p_{2}^{r_{2}}\right)$;

(iii) there is a positive integer $t$ such that $p_{2}^{t} \equiv-1\left(\bmod p_{1}^{r_{1}}\right)$.

Let $m$ be the smallest odd positive integer such that $x^{2}+p y^{2}=2^{m+2}$ has integral solution $(x, y)$. Then there is no GBFs of type $[\mathrm{m}, 2 \mathrm{~N}]$.

The above case is different from the ones in Feng et al. 4, 5, 3, 11, so the result is new. See Remark 4.6 for details.

And we also obtain the non-existence results of two types $\left[1 \leq n \leq 3,2 \times 31^{e}\right]$ and $[1 \leq n \leq$ $\left.7,2 \times 151^{e}\right]:$

Theorem 1.5. Let $e$ and $n$ be positive integers and $n$ be odd. Then there is no GBFs of type $\left[1 \leq n \leq 3,2 \times 31^{e}\right]$ and $\left[1 \leq n \leq 7,2 \times 151^{e}\right]$.

Remark 1.6. The result in [4, Theorem 3.1] can only deal with types $\left[n<m / s, p^{l}\right]$ where $s=\operatorname{ord}_{p^{l}}(2)$ and $p \equiv 7(\bmod 8)$. By the calculation in $4, \mathrm{pp} .566], \mathrm{m} / \mathrm{s}=1,7 / 5$ for $p=31,151$. Thus the results in the above theorem is also new.

For the proofs of the two theorems, we need some facts in algebraic number theory which are contained in Section 2. And for the cases where (1.3) is solvable over $\mathbb{Z}\left[\zeta_{q}\right]$, we generalize the idea used in 15, 7, 8, 10, which we name as the element partition method and prove in Section 3. With these preparations, we can prove Theorem 1.4 and 1.5 in Section 4 and 5 , respectively. Finally, we give some additional non-existence results obtained by PARI/GP [18 without proofs, and some remarks concerning the future work (Section 6). 


\section{BASIC FACTS OF ALGEBRAIC NUMBER THEORY}

The methods of proving non-existence results of GBF are often involve algebraic number theory, mainly the basic arithmetic (ideals, units, class groups etc.) of cyclotimic fields and their subfields. The standard reference is [12, Chapter 2,3] or [17, Chapter 2]. In this section, we list some facts needed in the proof.

For any number field $F$, denote by $\mathfrak{o}_{F}$ the ring of integers of $F$, by $C l(F)$ the class group of $F$ and by $h(F)$ the class number of $F$.

Let $h_{N}=h\left(\mathbb{Q}\left(\zeta_{N}\right)\right)$ and $h_{N}^{+}=h\left(\mathbb{Q}\left(\zeta_{N}+\zeta_{N}^{-1}\right)\right)$. It is well-known that $h_{N}^{+} \mid h_{N}$ and thus one can write $h_{N}=h_{N}^{+} h_{N}^{-}$.

For the subfield of the cyclotomic field $\mathbb{Q}\left(\zeta_{p^{e}}\right)$ where $p^{e}$ is a prime power, we have the divisibility of class numbers.

Lemma 2.1. Let $L=\mathbb{Q}\left(\zeta_{p^{e}}\right)$. Let $F$ be a subfield of $L$. Then we have $h(F) \mid h(L)$.

Proof. Let $H_{F}$ (resp. $H_{L}$ ) be the Hilbert class field of $F$ (resp. $L$ ). Then $H_{F} / F$ is unramified abelian, so the same is true for $L H_{F} / L$. It follows that $L H_{F} \subseteq H_{L}$. But $H_{F} \cap L=F$ in our case since $p$ is totally ramified in $L$. Hence

$$
h(F)=\left[H_{F}: F\right]=\left[L H_{F}: L\right] \mid\left[H_{L}: L\right]=h(L) .
$$

For more specific cases, we have the more strong

Proposition 2.2. Let $p \equiv 3(\bmod 4)$ and $L=\mathbb{Q}\left(\zeta_{p^{e}}\right), F:=\mathbb{Q}(\sqrt{-p})$. It is well-known that $F$ is a subfield of $L$. Let $E$ be any number field such that $F \subseteq E \subseteq L$. Then the canonical morphism $j_{E / F}: C l(F) \longrightarrow C l(E)$ sending $\mathfrak{a}$ to $\mathfrak{a o}_{E}$ is injective.

Proof. Let $I_{F}$ be the group of fractional ideals of $F$, and $P_{F}$ be principal ones. Let $I_{E}$ and $P_{E}$ be the corresponding groups for $E$. We know the Galois group $G:=\operatorname{Gal}(E / F)$ acts on $P_{E}$ and $\mathfrak{o}_{E}^{\times}$. Then by Greenberg [6, Proposition 1.2.3] we have

$$
\operatorname{ker}\left(j_{E / F}\right) \hookrightarrow P_{E}^{G} / P_{F} \cong H^{1}\left(G, \mathfrak{o}_{F}^{\times}\right)
$$

Let $n=[E: F]$ then $n \mid \varphi\left(p^{e}\right) / 2$ is odd since $p \equiv 3(\bmod 4)$. Thus

$$
\mathfrak{o}_{F}^{\times}=\{ \pm 1\}=N_{E / F}( \pm 1) \subseteq N_{E / F}\left(\mathfrak{o}_{E}^{\times}\right) .
$$

Note $E / F$ is cyclic. Thus we have $H^{2}\left(G, \mathfrak{o}_{E}^{\times}\right)=H^{0}\left(G, \mathfrak{o}_{E}^{\times}\right)$is trivial.

On the other hand, Greenberg [6. Proposition 1.2.4] tells us that

$$
\# H^{1}\left(G, \mathfrak{o}_{E}^{\times}\right)=n \# H^{2}\left(G, \mathfrak{o}_{E}^{\times}\right) .
$$

Combining with (2.3) we know

$$
\#\left(P_{E}^{G} / P_{F}\right)=n
$$

Let $\mathfrak{P}$ be a prime of $E$ dividing $p$ and $\mathfrak{p}=\mathfrak{P} \cap F$. Since $p$ is totally ramified in $L \supseteq E$ we know $\mathfrak{P} \in P_{E}^{G} / P_{F}$.

Now we claim that $\mathfrak{P}$ has order $n$ and hence $P_{E}^{G} / P_{F}$ is generated by $\mathfrak{P}$. Actually, $\mathfrak{P}^{n}=\mathfrak{p o}_{E} \in P_{F}$.

On the other hand, if we have $\mathfrak{P}^{k} \in P_{F}$ for some $k$, then $\mathfrak{P}^{k}=\alpha \mathfrak{o}_{E}$ for some $\alpha \in F$. It follows that

$$
\operatorname{ord}_{\mathfrak{p}}(\alpha) n=\operatorname{ord}_{\mathfrak{P}}(\alpha)=k .
$$

Hence $n \bmod k$. This proves the claim and that $P_{E}^{G} / P_{F}$ is generated by $\mathfrak{P}$. 
Now if $\mathfrak{a} \in \operatorname{ker}\left(j_{E / F}\right)$ then $\mathfrak{a o}_{E}=\beta \mathfrak{o}_{E}$ for some $\beta \in E$. Consider its image in $P_{E}^{G} / P_{F}=\langle\mathfrak{P}\rangle$ so we have $\mathfrak{a o}_{E}=\mathfrak{P}^{l} \gamma$ for some $l$ and $\gamma \in F$. Again the fact that

$$
\operatorname{ord}_{\mathfrak{p}}(\mathfrak{a} / \gamma) n=\operatorname{ord}_{\mathfrak{P}}\left((\mathfrak{a} / \gamma) \mathfrak{o}_{E}\right)=l
$$

gives $n \mid l$, which is to say $\mathfrak{a}$ is trivial in $P_{E}^{G} / P_{F}$. Then the injection in (2.3) implies that $\operatorname{ker}\left(j_{E / F}\right)$ is trivial and we complete the proof of the proposition.

Next we introduce Stickelberger ideals. Suppose $p$ is a prime and $K_{0}=\mathbb{Q}\left(\zeta_{p}\right)$ and $G_{0}=$ $\operatorname{Gal}\left(K_{0} / \mathbb{Q}\right) \cong(\mathbb{Z} / p \mathbb{Z})^{\times}$.

Definition 2.4. The Stickelberger element $\theta=\theta_{p} \in \mathbb{Q}\left[G_{0}\right]$ is defined by

$$
\theta=\sum a \in(\mathbb{Z} / p \mathbb{Z})^{\times}\left\{\frac{a}{p}\right\} \sigma_{a}^{-1}
$$

where $\left\{\frac{a}{p}\right\}=\frac{a}{p}-p\left[\frac{a}{p}\right]$. And the Stickelberger ideal $S_{p}$ of $\mathbb{Z}\left[G_{0}\right]$ is defined by

$$
S_{p}=\mathbb{Z}\left[G_{0}\right] \theta \cap \mathbb{Z}\left[G_{0}\right] .
$$

We mainly use these following properties of Stickelberger ideal:

Proposition 2.5. We have

(a) For $(c, p)=1$, the element $\left(c-\sigma_{c}\right) \theta$ are in $S_{p}$.

(b) The Stickelberger ideal $S_{p}$ annihilates the ideal class group $C l(M)$, where $M$ is a subfield of $K_{0}$ such that $p$ is the minimal integer with the property that $M \subseteq \mathbb{Q}\left(\zeta_{p}\right)$.

Proof. See [17, Lemma 6.9 and Theorem 6.10].

Through this paper, we fix the following notations. Let $q=2 N$ with $2 \nmid N \geq 3, \zeta=\zeta_{N}$ be an $N$-th primitive root of unity, $K=\mathbb{Q}(\zeta)$, and $D \subset K$ be the decomposition field of 2 in $K$. Let $G=\operatorname{Gal}(K / \mathbb{Q})$. It's well-known that $G \cong(\mathbb{Z} / N \mathbb{Z})^{\times}$, the isomorphism being $c \mapsto\left(\sigma_{c}: \zeta \mapsto \zeta^{c}\right)$ for $\operatorname{gcd}(c, N)=1$.

For our purpose we need to investigate the equation $\alpha \bar{\alpha}=q^{n}=(2 N)^{n}$ where $\alpha \in \mathbb{Z}\left[\zeta_{N}\right]$. So we first study the idealic behaviour of 2 and $p$ in the cyclotomic field $K$.

The following lemma taken from Feng [4, Lemma 2.1] will descent the equation $\alpha \bar{\alpha}=2^{n}, \alpha \in \mathfrak{o}_{K}$ to $\alpha \bar{\alpha}=2^{n}, \alpha \in \mathfrak{o}_{E}$, where $E$ is a subfield of $K$.

Lemma 2.6 (4], Lemma 2.1). If $\alpha \bar{\alpha}=2^{n}$ for some $\alpha \in \mathfrak{o}_{K}$ and a positive integer $n$, then there exist $\beta= \pm \zeta^{j} \alpha$ for some $j \in \mathbb{Z}$ and subfield $E \subseteq K$ containing $D$ with $[E: D] \leq 2$ such that $\beta \in \mathfrak{o}_{E}$ and $\beta \bar{\beta}=2^{n}$.

\section{The ElEment PARTition Method}

In this section, we will prove the following

Proposition 3.1. Suppose $t$ is an odd positive integer and $q=2 N, 2 \nmid N \geq 3$. Let $f: \mathbb{Z}_{q}^{t} \longrightarrow \mathbb{Z}_{q}$ be a function with $F(\lambda)$ its Fourier transform defined as before. Suppose $F(\lambda)$ has the property that for every $\lambda \in \mathbb{Z}_{q}^{t}$ and $v \in \mathbb{Z}_{q}^{t}$ an element of order 2 ,

$$
\begin{gathered}
F(\lambda) \nsubseteq 2 \mathfrak{o}_{K} \\
\text { and } F(\lambda) \mathfrak{o}_{K}=F(\lambda+v) \mathfrak{o}_{K} .
\end{gathered}
$$

Then $f$ is not a GBF. 
The idea behind this proposition dates back to the method developed by Ikeda [7] and Jiang-Deng 8]. To prove the proposition we have to generalize this.

Now we assume $f$ is a GBF so by definition we have $F(\lambda) \overline{F(\lambda)}=q^{n}$, which leads to the following

Lemma 3.4. Let $\lambda, v, f, F$ be as in Proposition 3.1, If $f$ is a $G B F$, we have $F(\lambda)= \pm F(\lambda+v)$.

Proof. Since $v$ is of order 2 , we have $\zeta_{q}^{x \cdot v}= \pm 1$. So

$$
F(\lambda)+F(\lambda+v)=\sum_{x \in \mathbb{Z}_{q}^{t}} \zeta_{q}^{f(x)-x \cdot \lambda}\left(1+\zeta_{q}^{-x \cdot v}\right) \in 2 \mathfrak{o}_{K} .
$$

From (3.3) we know that $u:=F(\lambda) / F(\lambda+v) \in \mathfrak{o}_{K}^{\times}$. For each $\sigma \in G$,

$$
\left.\sigma(u) \overline{\sigma(u)}=\sigma(u \bar{u})=\sigma\left(\frac{F(\lambda) \overline{F(\lambda)}}{F(\lambda+v) \overline{F(\lambda+v)}}\right)=1, \quad \text { (by (1.2) }\right)
$$

whence $u$ is a root of unity and so $u= \pm \zeta^{j}$. Thus

$$
F(\lambda)= \pm \zeta^{j} F(\lambda+v)
$$

Then

$$
F(\lambda)+F(\lambda+v)=F(\lambda)\left(1 \pm \zeta^{j}\right) .
$$

It follow from (3.5) that $2 \mid F(\lambda)\left(1 \pm \zeta^{j}\right)$ in $\mathfrak{o}_{K}$. Note that if $\zeta^{j} \neq 1$ then $1 \pm \zeta^{j}$ is an unit or divides $N$, so $2 \mathfrak{o}_{K}+\left(1 \pm \zeta^{j}\right) \mathfrak{o}_{K}=\mathfrak{o}_{K}$. Thus $2 \mid F(\lambda)$, which is a contradiction to the hypothesis (3.2). This shows that $F(\lambda)= \pm F(\lambda+v)$.

Now let us pin down some notations. Let $P_{2}$ be the Sylow-2 subgroup of $\mathbb{Z}_{q}^{t}$. Then $P_{2} \cong \mathbb{F}_{2}^{t}$ as $\mathbb{F}_{2}$-vector space and we write $P_{2}=\left\{0, v_{1}, \ldots, v_{2^{t}-1}\right\}$. For every $v_{i} \in P_{2}$, we know that $v_{i}$ has order 2. If we define

$$
\begin{gathered}
N_{i}=N_{v_{i}}=\left\{x \in \mathbb{Z}_{q}^{t} \mid F(x)=F\left(x+v_{i}\right)\right\}, \\
\text { and } M_{i}=M_{v_{i}}:=\left\{x \in \mathbb{Z}_{q}^{t} \mid F(x)=-F\left(x+v_{i}\right)\right\},
\end{gathered}
$$

then $\mathbb{Z}_{q}^{t}=N_{i} \sqcup M_{i}$ by Lemma (3.4), where the symbol $\sqcup$ means disjoint union.

Remark 3.6. For sake of the above decomposition $\mathbb{Z}_{q}^{t}=N_{i} \sqcup M_{i}$, we call this method element partition.

Let $n_{i}=\# N_{i}, m_{i}=\# M_{i}$ be the cardinality of $N_{i}, M_{i}$, so $n_{i}+m_{i}=q^{t}$.

Lemma 3.7. $n_{i}=m_{i}=\frac{q^{t}}{2}$.

Proof. As $F$ is a Fourier transform of $\zeta^{f(x)}$, which is a function from $\mathbb{Z}_{q}^{t}$ to $\mathbf{S}^{1}=\{z \in \mathbb{C}|| z \mid=1\}$, we have

$$
\sum_{x \in \mathbb{Z}_{q}^{t}} F(x) \overline{F\left(x+v_{i}\right)}=0 .
$$

Then by the definition (1.2), $F(x) \overline{F(x)}=q^{t}$ for each $x$. It follows that

$$
0=\sum_{x \in N_{i}} F(x) \overline{F\left(x+v_{i}\right)}+\sum_{x \in M_{i}} F(x) \overline{F\left(x+v_{i}\right)}=q^{t}\left(n_{i}-m_{i}\right) .
$$

Hence, $n_{i}=m_{i}$ for each $i$. 
Here we review the proof of Ikeda [7] for the case $t=1$, since it is the basic idea of this method. First note that $x \in N_{v}$ if and only if $x+v \in N_{v}$, where $v$ is the unique element of order 2 in $\mathbb{Z}_{q}$. Hence $2 \mid n_{v}$. But $n_{v}=\frac{q}{2}$ is odd, which is a contradiction.

It can be seen that Ikeda 7] used one 2-order element to prove the result in the case $t=1$. In Jiang-Deng [8], they use three 2-order elements to treat the case $t=3$.

We now generalize this method systematically to treat the general case. The second author used the same augment in $\left[10\right.$ to prove that there is no GBF of type $\left[m, 2 p^{e}\right]$ where $p \equiv 7(\bmod 8)$ is a prime with $\operatorname{ord}_{p^{e}}(2)=\varphi\left(p^{e} / 2\right)$ and $m$ is defined the same as in Theorem 1.4.

In the remain of the proof, we assume $t \geq 3$ so that $\# P_{2} \geq 8$. Now we define $2^{2^{t}-1}$ subsets of $\mathbb{Z}_{q}^{t}$ by using all 2-order elements as follows:

$$
X_{1} \cap X_{2} \cap \cdots \cap X_{2^{t}-1},
$$

with each $X_{i}=N_{i}$ or $M_{i}$. Obviously, $\mathbb{Z}_{q}^{t}$ is a disjoint union of all these subsets. Our main task is to compute the cardinality of each subsets.

Lemma 3.8. If $u, v, w \in P_{2}-\{0\}$ are pairwise different and $u+v+w=0$, then we have

$$
N_{u} \cap N_{v} \cap M_{w}=M_{u} \cap M_{v} \cap M_{w}=\emptyset .
$$

Proof. We only need the assumption (3.2) that $F(\lambda) \notin 2 \mathfrak{o}_{K}$, so the proof below is essentially same as the case $p=23$ as in [8, Lemma 11].

First, note that

$$
\begin{aligned}
x & \in N_{u} \cap N_{v} \cap M_{w} \\
\Longleftrightarrow x+w & \in M_{u} \cap M_{v} \cap M_{w} .
\end{aligned}
$$

So it's enough to prove $M_{u} \cap M_{v} \cap M_{w}=\emptyset$.

Second, note that the map

$$
y \mapsto y \cdot u: \mathbb{Z}_{q}^{t} \longrightarrow\left\{0, \frac{q}{2}\right\} \subset \mathbb{Z}_{q}
$$

is surjective, so

$$
\zeta^{y \cdot u}= \begin{cases}1 & \text { if } y \cdot u=0 \\ -1 & \text { if } y \cdot u \neq 0\end{cases}
$$

For simplicity, we write $\sum=\sum \zeta^{f(y)-x \cdot y}$. Now take an element $x \in M_{u} \cap M_{v} \cap M_{w}$, then

$$
\begin{aligned}
F(x) & =\sum_{y \in \mathbb{Z}_{q}^{t}}=\sum_{y \cdot u=0}+\sum_{y \cdot u \neq 0} \\
& =-F(x+u)=-\sum_{y \cdot u=0}+\sum_{y \cdot u \neq 0} .
\end{aligned}
$$

So we get

$$
0=\sum_{y \cdot u=0}=\sum_{y \cdot u=0, y \cdot v=0}+\sum_{y \cdot u=0, y \cdot v \neq 0} .
$$

Similarly, we have

$$
0=\sum_{y \cdot v=0}=\sum_{y \cdot u=0, y \cdot v=0}+\sum_{y \cdot u \neq 0, y \cdot v=0} .
$$

Also since

$$
0=\sum_{y \cdot w=0}
$$


we have that

$$
F(x)=\sum_{y \cdot w \neq 0}=\sum_{y \cdot(u+v) \neq 0}=\sum_{y \cdot u=0, y \cdot v \neq 0}+\sum_{y \cdot u \neq 0, y \cdot v=0}=-2 \sum_{y \cdot u=0, y \cdot v=0} \in 2 \mathfrak{o}_{K} .
$$

This contradict to (3.2). So $M_{u} \cap M_{v} \cap M_{w}=\emptyset$.

Then we have the following observation. It tells us among the $2^{2^{t}-1}$ subsets, there are at most $2^{t}$ nonempty subsets. And these $2^{t}$ subsets are rather "nice".

Lemma 3.9. Let $N_{i}, M_{i}$ be as above, $X_{1} \cap X_{2} \cap \cdots \cap X_{2^{t}-1} \subseteq \mathbb{Z}_{q}^{t}$ with $X_{i}=N_{i}$ or $M_{i}$. If $\left\{v_{i} \in P_{2} \mid X_{i}=N_{i}\right\} \cup\{0\}$ is not a subgroup of $P_{2}$ with index 1 or 2 , then it must be empty.

Proof. If $A:=\left\{v_{i} \in P_{2} \mid X_{i}=N_{i}\right\} \cup\{0\}$ is not a subgroup, then there are $u, v \in A-\{0\}$ such that $u+v \notin A$, hence $X_{u}=N_{u}, X_{v}=N_{v}$ and $X_{u+v}=M_{u+v}$. Then by Lemma 3.8.

$$
X_{1} \cap X_{2} \cap \cdots \cap X_{2^{t}-1} \subseteq N_{u} \cap N_{v} \cap M_{u+v}=\emptyset .
$$

If $A$ is a subgroup with index larger than 2. Then $A$ is also a $\mathbb{F}_{2}$ vector subspace of $P_{2}$ and its $\mathbb{F}_{2}$-dimension is less than or equal to $t-2$. Thus the dimension of its complement subspace $\bar{A}$ is greater than or equal to 2 , so we can take $u, v \in \bar{A}$ such that they are independent. Then $u, v, u+v \in \bar{A}-\{0\}$, so $u, v, u+v$ are not in $A$ and hence $X_{u}=M_{u}, X_{v}=M_{v}$ and $X_{u+v}=M_{u+v}$. Then by Lemma 3.8 we have

$$
X_{1} \cap X_{2} \cap \cdots \cap X_{2^{t}-1} \subseteq M_{u} \cap M_{v} \cap M_{u+v}=\emptyset
$$

and we finish the proof.

The following lemma is a basic fact about the subgroups of $P_{2}$.

Lemma 3.10. There are $2^{t}-1$ subgroups of $P_{2}$ with index 2 .

Proof. The correspondence

$$
\begin{gathered}
\text { subgroups of index } 2 \\
=\left\{\mathbb{F}_{2} \text { vector subspace of } P_{2} \text { with dimension } t-1\right\} \\
\longleftrightarrow\left\{\mathbb{F}_{2} \text { vector subspace with dimension } 1\right\}
\end{gathered}
$$

is one-to-one by taking the complement subspace. Since there are $2^{t}-1$ nonzero elements in $P_{2}$, there are $2^{t}-1$ subgroups with index 2 by the above correspondence.

Let $\left\{\right.$ subgroups of $P_{2}$ with index 2$\}=\left\{H_{1}, \ldots, H_{2^{t}-1}\right\}$. Among the $2^{2^{t}-1}$ subsets $X_{1} \cap X_{2} \cap$ $\cdots \cap X_{2^{t}-1}$ with $X_{i}=N_{i}$ or $M_{i}$, we only need to consider the following subsets, since others are empty sets by Lemma 3.9.

$$
\begin{aligned}
& Y_{0}:=\bigcap_{i=1}^{2^{t}-1} N_{i} \\
& \text { (corresponding to the subgroup with index 1) } \\
& \text { and } Y_{i}:=\left(\bigcap_{v \in H_{i}-\{0\}} N_{v}\right) \bigcap\left(\bigcap_{u \in P_{2}-H_{i}} M_{u}\right) \text {, } \\
& i=1,2, \ldots, 2^{t}-1 \text {. } \\
& \text { (corresponding to subgroups with index 2) }
\end{aligned}
$$

Let $y_{0}=\# Y_{0}$ and $y_{i}=\# Y_{i}$ for $i=1,2, \ldots, 2^{t}-1$. 
Proof of the Proposition 3.1. Recall that $\mathbb{Z}_{q}^{t}$ is a disjoint union of the following $2^{2^{t}-1}$ subsets $X_{1} \cap$ $X_{2} \cap \cdots \cap X_{2^{t}-1}$ where each $X_{i}=N_{i}$ or $M_{i}$. Since the only possibly nonempty subsets are $Y_{0}, \ldots, Y_{2^{t}-1}$ by Lemma 3.9 and Lemma 3.10, we have

$$
\mathbb{Z}_{q}^{t}=\bigsqcup_{i=0}^{2^{t}-1} Y_{i}
$$

from which we obtain an equation

$$
y_{0}+\sum_{i=1}^{2^{t}-1} y_{i}=q^{t}
$$

On the other hand

$$
\begin{aligned}
N_{i} & =N_{i} \cap \mathbb{Z}_{q}^{t}=N_{i} \bigcap\left(\bigsqcup_{i=0}^{2^{t}-1} Y_{i}\right) \\
& =Y_{0} \bigcup\left(\bigsqcup_{1 \leq j \leq 2^{t}-1, v_{i} \in H_{j}} Y_{j}\right), \quad i=1, \ldots, 2^{t}-1 .
\end{aligned}
$$

Note that $n_{i}=\frac{q_{t}}{2}$ by Lemma 3.7. We obtain

$$
\frac{q^{t}}{2}=n_{i}=y_{0}+\sum_{1 \leq j \leq 2^{t}-1, v_{i} \in H_{j}} y_{j}, \quad i=1, \ldots, 2^{t}-1 .
$$

By summing up the above $2^{t}-1$ equations, we obtain

$$
\begin{aligned}
\left(2^{t}-1\right) \frac{q^{t}}{2} & =\sum_{i=1}^{2^{t}-1}\left(y_{0}+\sum_{1 \leq j \leq 2^{t}-1, v_{i} \in H_{j}} y_{j}\right) \\
& =\left(2^{t}-1\right) y_{0}+\sum_{j=1}^{2^{t}-1} y_{j} \sum_{1 \leq i \leq 2^{t}-1, v_{i} \in H_{j}} 1 \\
& =\left(2^{t}-1\right) y_{0}+\sum_{j=1}^{2^{t}-1} y_{j} \#\left(H_{j}-\{0\}\right) \\
& =\left(2^{t}-1\right) y_{0}+\left(2^{t-1}-1\right) \sum_{i=1}^{2^{t}-1} y_{i} . \quad\left(\text { since } H_{j} \subset P_{2}\right. \text { with index 2) }
\end{aligned}
$$

Conbining with (3.11), we treat them as two linear equations with two unknown variables $y_{0}$ and $\sum_{i=1}^{2^{t}-1} y_{i}$. Soving the equation we have $y_{0}=\frac{q^{t}}{2^{t}}=N^{t}$ is an odd number.

However, fixing any $v \in P_{2}-\{0\}$, we have $x \in Y_{0}$ if and only if $x+v \in Y_{0}$, so $2 \mid y_{0}$. This contradiction shows that $f$ is not a GBF.

\section{Non-existence Result for GBFs of the type $\left[m, 2 p_{1}^{r_{1}} p_{2}^{r_{2}}\right]$}

In this section, we will prove Theorem 1.4, where $N=p_{1}^{r_{1}} p_{2}^{r_{2}}$ satisfy (i), (ii) and (iii). Assume $f$ is a GBF of type $[n=m, q=2 N]$. Then $F(\lambda) \in \mathfrak{o}_{K}$ and $F(\lambda) \overline{F(\lambda)}=(2 N)^{m}$. Let $K=\mathbb{Q}\left(\zeta_{N}\right)$ and $D$ be the decomposition group of 2 in $K$ as before. By hypothesis (i) we know that $[D: \mathbb{Q}]=2$. 
While $p \equiv 7(\bmod 8)$ implies that 2 splits in $\mathbb{Q}(\sqrt{-p})$. Hence $D=\mathbb{Q}(\sqrt{-p})$ is the decomposition group of 2 in $K$. We now have the following statement about the integer $m$.

Lemma 4.1. Let $E$ be any extension of $D$ with $[E: D] \leq 2$ Let $\mathfrak{P}$ be a prime in $E$ lying over 2 . Then the odd integer $m$ defined in Theorem 1.4 is equal to the order of $\mathfrak{P}$ in the class group $C l(E)$.

Proof. Let $\mathfrak{p}=\mathfrak{P} \cap D$ and denote by $a_{p}$ (resp. $b_{p}$ ) the order of $\mathfrak{p}$ in $C l(D)$ (resp. of $\mathfrak{P}$ in $C l(E)$ ). By Gauss' genus theory or class field theory [17, Theorem 10.4(b)], $C l(D)$ is odd. So is $a_{p}$. Remember $D=\mathbb{Q}(\sqrt{-p})$ and $2 \mathfrak{o}_{D}=\mathfrak{p} \overline{\mathfrak{p}}$ splits, so $\mathfrak{p}^{a_{p}}=(A+B \sqrt{-p}) / 2$ is principal in $\mathfrak{o}_{D}$ for some integers $A$ and $B$. It follows that $2^{a_{p}} \mathfrak{o}_{D}=\mathfrak{p}^{a_{p}} \overline{\mathfrak{p}}^{a_{p}}=\frac{A^{2}+p B^{2}}{4} \mathfrak{o}_{D}$ and thus $A^{2}+p B^{2}=2^{a_{p}+2}$, which implies $m \leq a_{p}$ since $a_{p}$ is odd.

On the other hand, suppose the equation $x^{2}+p y^{2}=2^{m+2}$ has integral solution $(A, B)$. We know that both $A$ and $B$ should be odd. Thus let $\delta=(A+B \sqrt{-p}) / 2 \in \mathfrak{o}_{D}$, we have $\delta \bar{\delta} \mathfrak{o}_{D}=2^{m} \mathfrak{o}_{D}=$ $\mathfrak{p}^{m} \overline{\mathfrak{p}}^{m}$. Since $m \leq a_{p}$ and $a_{p}$ is the smallest integer such that $\mathfrak{p}_{p}^{a}$ is principal, we have $m=a_{p}$.

Now we observe that $C l(D) \longrightarrow C l(E)$ is injective by Proposition 2.2 Thus $a_{p}=b_{p}$ and the result follows.

Let $\mathfrak{P}$ be as in Lemma 4.1. Then $\mathscr{P}:=\mathfrak{P o}_{K}$ is a prime ideal of $K$ since $D$ is the decomposition field. Also $2 \mathfrak{o}_{K}=\mathscr{P} \overline{\mathscr{P}}$. We use the following lemma to characterize $F(\lambda)$.

Lemma 4.2. Suppose $f$ is a GBF of type $\left[m, q=2 p_{1}^{r_{1}} p_{2}^{r_{2}}\right]$ where $m, p_{1}, p_{2}, r_{1}, r_{2}$ is defined as in Theorem 1.4. Then there exists an $o_{K}$-ideal $\mathscr{A}$ not divisible by $\mathscr{P}$ nor $\mathscr{P}$, such that for each $\lambda \in \mathbb{Z}_{q}^{m}$, we have $F(\lambda) \mathfrak{o}_{K}=\mathscr{A}^{m}$ or $\mathscr{A} \overline{\mathscr{P}}^{m}$.

Proof. Since $f$ is a GBF of type $\left[m, q=2 p_{1}^{r_{1}} p_{2}^{r_{2}}\right]$, we have

$$
F(\lambda) \overline{F(\lambda)}=\left(2 p_{1}^{r_{1}} p_{2}^{r_{2}}\right)^{m} \text {. }
$$

By hypothesis (ii) we known that $\operatorname{ord}_{p_{2}}{ }_{2}\left(p_{1}\right)$ is odd and thus the complex conjugation is in the decomposition group of $p_{1}$ in $K$. This implies that every prime in $K$ lying over $p_{1}$ is fixed by the complex conjugation. We also observe that $\sqrt{-p_{1}} \in \mathfrak{o}_{K}$ and $\left(\sqrt{-p_{1}}\right)^{2} \mathfrak{o}_{K}=p_{1} \mathfrak{o}_{K}$. By consider the order of each prime in $K$ lying over $p_{1}$ appearing in the equation (4.3), we known that $F(\lambda) /\left(\sqrt{-p_{1}}\right)^{m r_{1}} \in \mathfrak{o}_{K}$. And similarly by hypothesis (iii), $F(\lambda) /\left(\sqrt{p_{2}}\right)^{m r_{2}} \in \mathfrak{o}_{K}$. So if we set

$$
\alpha=\frac{F(\lambda)}{\left(\sqrt{-p_{1}}\right)^{m r_{1}}\left(\sqrt{p_{2}}\right)^{m r_{2}}},
$$

then $\alpha \in \mathfrak{o}_{K}$ and $\alpha \bar{\alpha}=2^{m}$. Now we use Lemma 2.6 to obtain $\beta \in \mathfrak{o}_{E}$ and $\beta \bar{\beta}=2^{m}$ for some $\beta= \pm \zeta^{j} \alpha \in \mathfrak{o}_{E}$ and $j \in \mathbb{Z}$, where $E$ is a subfield of $K$ containing $D$ with $[E: D] \leq 2$.

Recall that $\operatorname{ord}_{N}(2)=\varphi(N) / 2$ and $D \subseteq E$, so $2 \mathfrak{o}_{E}=\mathfrak{P} \bar{P}$ is the prime decomposition of 2 in $E$. Then we have $\beta \bar{\beta} \mathfrak{o}_{E}=\mathfrak{P}^{m} \overline{\mathfrak{P}}^{m}$. Note that $m$ is the order of $\mathfrak{P}$ in $C l(E)$ by Lemma 4.1, so we know $\beta \mathfrak{o}_{E}=\mathfrak{P}^{m}$ or $\overline{\mathfrak{P}}^{m}$. By (4.4) we have

$$
F(\lambda)=\alpha\left(\sqrt{-p_{1}}\right)^{m r_{1}}\left(\sqrt{p_{2}}\right)^{m r_{2}}= \pm \beta \zeta^{j}\left(\sqrt{-p_{1}}\right)^{m r_{1}}\left(\sqrt{p_{2}}\right)^{m r_{2}} .
$$

Hence

$$
\left.F(\lambda) \mathfrak{o}_{K}=\mathfrak{P}^{m}\left(\sqrt{-p_{1}}\right)^{m r_{1}}\left(\sqrt{p_{2}}\right)^{m r_{2}} \mathfrak{o}_{K}=\mathscr{A}^{m} \text { (or } \mathscr{A} \overline{\mathscr{P}}^{m}\right),
$$

where $\mathscr{A}:=\left(\sqrt{-p_{1}}\right)^{m r_{1}}\left(\sqrt{p_{2}}\right)^{m r_{2}} \mathfrak{o}_{K}$ is clearly not divisible by $\mathscr{P}$ nor $\overline{\mathscr{P}}$. So we complete the our proof. 
Remark 4.6. From (4.5) we can easily verify that such $F(\lambda)$ satisfy the equation

$$
F(\lambda) \overline{F(\lambda)}=q^{n} \text {. }
$$

So the case here is different from Feng's [4, 5, 3] and hence the result stated in this theorem is new.

Now we turn to the

Proof of the Theorem 1.4. Let $m, q$ be as hypotheses. Let $f: \mathbb{Z}_{q}^{m} \longrightarrow \mathbb{Z}_{q}$ be a function with Fourier transform $F(\lambda)$. Our task is to establish the property (3.2) and (3.3) of $F(\lambda)$ needed in Proposition 3.1. Suppose $\lambda \in \mathbb{Z}_{q}^{m}$ and $v \in \mathbb{Z}_{q}^{m}$ an element of order 2, we have $\zeta_{q}^{x \cdot v}= \pm 1$, so

$$
F(\lambda)+F(\lambda+v)=\sum_{x \in \mathbb{Z}_{q}^{t}} \zeta_{q}^{f(x)-x \cdot \lambda}\left(1+\zeta_{q}^{-x \cdot v}\right) \in 2 \mathfrak{o}_{K} .
$$

Lemma 4.2 tells us that $F(\lambda) \mathfrak{o}_{K}$ and $F(\lambda+v) \mathfrak{o}_{K}$ can only be one of the two ideals $\mathscr{A} \mathscr{P}^{m}$ and $\mathscr{A} \overline{\mathscr{P}}^{m}$ where $\mathscr{A}$ is not divisible by $\mathscr{P}$ nor $\overline{\mathscr{P}}$. In particular, $F(\lambda) \mathfrak{o}_{K} \not \subseteq 2 \mathfrak{o}_{K}=\mathscr{P} \overline{\mathscr{P}}$ and (3.2) follows. If we assume $F(\lambda) \mathfrak{o}_{K}$ and $F(\lambda+v) \mathfrak{o}_{K}$ are different, say, $F(\lambda) \mathfrak{o}_{K}=\mathscr{A} \mathscr{P}^{m}$ and $F(\lambda+v) \mathfrak{o}_{K}=\mathscr{A} \overline{\mathscr{P}}^{m}$. Then (4.7) tells us $F(\lambda+v) \mathfrak{o}_{K} \in \mathscr{P}$ since $2 \mathfrak{o}_{K} \in \mathscr{P}$. This contradicts to the assumption that $F(\lambda+v) \mathfrak{o}_{K}=\mathscr{A} \overline{\mathscr{P}}^{m}$. Hence $F(\lambda) \mathfrak{o}_{K}=F(\lambda+v) \mathfrak{o}_{K}=\mathscr{A}^{m}$. This proves (3.3).

By applying Proposition 3.1 we know that such function $f$ is not a GBF and we finish the proof.

\section{Non-existence Result for GBFs of the type $\left[n, 2 p^{e}\right]$ FOR CERTAin $n$ And $p$}

We will prove Theorem 1.5 in this section. First we fix some additional notations. Suppose $n$ is odd, $N=p^{e}$ where $p \equiv 7(\bmod 8)$ is a prime. Let $K=\mathbb{Q}\left(\zeta_{N}\right)$ and $D \subset K$ be the decomposition field of 2 in $K$ as before. Since $\left(\frac{2}{p}\right)=-1, f=\operatorname{ord}_{N}(2)$ is odd. Thus $g:=\varphi\left(p^{e}\right) / f$ is even and we set $u=g / 2$. Suppose the prime decomposition of 2 in $D$ is

$$
2 \mathfrak{o}_{D}=\mathfrak{P}_{1} \mathfrak{P}_{2} \ldots \mathfrak{P}_{g} .
$$

If there are GBFs of type $[n, q=2 N]$ then $F(\lambda) \overline{F(\lambda)}=(2 N)^{n}$. As in the proof of Lemma 4.2, if we set $\alpha=\frac{F(\lambda)}{(\sqrt{-p})^{e m}}$ then $\alpha \in \mathfrak{o}_{K}$ and $\alpha \bar{\alpha}=2^{n}$. Apply Lemma 2.6 we descent the above equation to a subfield $E \subseteq K$ with $[E: D] \leq 2$. Note $[E: D]$ divides the odd number $f$ it follows that $E=D$. Thus we obtain $\beta \in \mathfrak{o}_{D}$ and $\beta \bar{\beta}=2^{n}$ for some $\beta= \pm \zeta^{j} \alpha \in \mathfrak{o}_{D}$ and $j \in \mathbb{Z}$.

Since $f$ is odd, the complex conjugation is not in the decomposition group of 2 . Thus we may assume $\mathfrak{P}_{u+k}=\overline{\mathfrak{P}}_{k}, k=1,2, \ldots, u$. Then we have

$$
\beta \bar{\beta} \mathfrak{o}_{D}=\prod_{k=1}^{u} \mathfrak{P}_{k}^{n} \overline{\mathfrak{P}}_{k}^{n} .
$$

So

$$
\beta \mathfrak{o}_{D}=\prod_{k=1}^{u} \mathfrak{P}_{k}^{n_{k}} \overline{\mathfrak{P}}_{k}^{\bar{n}_{k}}
$$

where $n_{k}, \bar{n}_{k}$ are nonnegative integer such that $n_{k}+\bar{n}_{k}=n$ for all $k=1,2, \ldots, u$. 
For convenience we write $x_{k}$ for $\mathfrak{P}_{k}$ in $C l(D)$ and view $C l(D)$ additively. Hence (5.2) becomes

$$
\begin{gathered}
\sum_{k=1}^{u}\left(n_{k} x_{k}+\bar{n}_{k} \bar{x}_{k}\right)=0 \\
\text { where } n_{k}+\bar{n}_{k}=n, k=1,2, \ldots, u .
\end{gathered}
$$

With the above notations, we prove the following

Proposition 5.5. Let $N=p^{e}$ where $e$ is a positive integer and $p \equiv 7(\bmod 8)$ is a prime. Let $n_{0}$ be the least odd integer such that (5.3) has nonnegative integral solution $\left(n_{1}, n_{2}, \ldots, n_{g}\right)$, where $n_{k}+\bar{n}_{k}=n_{0}$ and $n_{u+k}:=\bar{n}_{k}, k=1,2, \ldots, u$. If $n<n_{0}$ is a positive odd integer, then there is no GBFs of type $[n, 2 N]$.

Further more, if $n=n_{0}$ and for all corresponding solutions $\left(n_{1}, n_{2}, \ldots, n_{g}\right)$, the set

$$
Z_{p}\left(n_{1}, \ldots, n_{g}\right):=\left\{j=1,2, \ldots, g \mid n_{j}=0\right\}
$$

are nonempty and pairwise different, then there is also no GBFs of type $\left[n_{0}, 2 N\right]$.

Proof. The first assertion is trivially true since (1.3) is not solvable over $\mathfrak{o}_{K}$ by the previous augment. For the second one, we use the element partition method described in Section 3 , The augment is similar to the one in the proof of Theorem 1.4. Suppose $v$ is of order 2, we have

$$
F(\lambda)+F(\lambda+v)=\sum_{x \in \mathbb{Z}_{q}^{t}} \zeta_{q}^{f(x)-x \cdot \lambda}\left(1+\zeta_{q}^{-x \cdot v}\right) \in 2 \mathfrak{o}_{K} .
$$

We will show that $F(\lambda) \mathfrak{o}_{K}=F(\lambda+v) \mathfrak{o}_{K} \not 2 \mathfrak{o}_{K}$ and the proposition follows from Proposition 3.1 We know that

$$
F(\lambda) \mathfrak{o}_{K}= \pm \zeta^{j}(\sqrt{-p})^{e m} \prod_{k=1}^{g} \mathscr{P}_{k}^{n_{k}}
$$

where $\mathscr{P}_{k}:=\mathfrak{P}_{k} \mathfrak{o}_{K}$ is the prime in $K$ lying over $\mathfrak{P}_{k}$ and $\left(n_{1}, n_{2}, \ldots, n_{g}\right)$ is a solution of (5.3). The hypothesis that

$$
Z_{p}\left(n_{1}, \ldots, n_{g}\right)=\left\{j=1,2, \ldots, g \mid n_{j}=0\right\}
$$

is nonempty means that

$$
F(\lambda) \mathfrak{o}_{K} \not 2 \mathfrak{o}_{K}=\mathscr{P}_{1} \mathscr{P}_{2} \ldots \mathscr{P}_{g} .
$$

If we assume $F(\lambda) \mathfrak{o}_{K}$ and $F(\lambda+v) \mathfrak{o}_{K}$ are different, then the corresponding solutions of (5.3) are also different. Since by hypothesis the corresponding $Z_{p}$ 's are different, we can assume that $\mathscr{P}_{j} \mid F(\lambda) \mathfrak{o}_{K}$ and $\mathscr{P}_{j} \nmid F(\lambda+v) \mathfrak{o}_{K}$ for some $j$. Then the decomposition $2 \mathfrak{o}_{K}=\mathscr{P}_{1} \mathscr{P}_{2} \ldots \mathscr{P}_{g}$ and (5.6) tell us $F(\lambda+v) \mathfrak{o}_{K} \in \mathscr{P}_{j}$, which contradicts to the assumption that $\mathscr{P}_{j} \nmid F(\lambda+v) \mathfrak{o}_{K}$. Hence $F(\lambda) \mathfrak{o}_{K}=F(\lambda+v) \mathfrak{o}_{K}$ and the proposition follows.

The above proposition is not concrete. To obtain the non-existence results in Theorem 1.5 we have to exploit the relations between $x_{k}$ 's in $C l(D)$. By (5.1) we have

$$
\sum_{k=1}^{g} x_{k}=0 \text {. }
$$

We want to find more relations.

Let $K_{0}=\mathbb{Q}\left(\zeta_{p}\right)$ and $F=\mathbb{Q}(\sqrt{-p})$ so we know that $F \subseteq K_{0} \subseteq K$. Suppose further that

$$
2^{p-1} \not \equiv 1 \quad\left(\bmod p^{2}\right) \text {. }
$$


One can check that this implies

$$
\frac{\varphi(p)}{\operatorname{ord}_{p}(2)}=\frac{\varphi\left(p^{e}\right)}{\operatorname{ord}_{p^{e}}(2)}=g
$$

It follows that $D$ is also the decomposition group of 2 in $K_{0}$. Let $K_{0}^{+}=K_{0} \cap \mathbb{R}=\mathbb{Q}\left(\zeta_{p}+\zeta_{p}^{-1}\right)$. Then Miller's work on class number of $K_{0}^{+}$gives

Theorem 5.9 ([13, Theorem 1.1). The class number of $\mathbb{Q}\left(\zeta_{p}+\zeta_{p}^{-1}\right)$ is 1 if $p \leq 151$ is a prime.

From now on we suppose $p=31$ or 151 . Then $p \equiv 7(\bmod 8)$ and one can check that the equation (5.8) is true. Let $D^{+}=D \cap \mathbb{R} \subseteq K_{0}^{+}$. Then $D$ is a quadratic extension over $D^{+}$. By Miller's result and Lemma 2.1, we have $h\left(D^{+}\right)=h\left(K_{0}^{+}\right)=1$. Now $2 \mathfrak{o}_{D^{+}}=\mathfrak{p}_{1} \mathfrak{p}_{2} \ldots \mathfrak{p}_{u}$ where $\mathfrak{p}_{k} \mathfrak{o}_{D}=\mathfrak{P}_{k} \overline{\mathfrak{P}}_{k}$, and all $\mathfrak{p}_{k}$ is principal since $h\left(D^{+}\right)=1$. This implie the relations

$$
x_{k}+x_{u+k}=0, \quad k=1,2, \ldots, u .
$$

However, these relations above are not enough. We need the Stickelberger ideal introduced in Section 2, Let $\mathfrak{P}=\mathfrak{P}_{1}$ and correspondingly $x=x_{1}$. Let $c$ be a integer not divisible by $p$. Since it is well-known that $p$ is the minimal integer such that $F \subseteq \mathbb{Q}\left(\zeta_{p}\right)$, it follows that $p$ is also the minimal one such that $D \subseteq \mathbb{Q}\left(\zeta_{p}\right)$. By Proposition 2.5, we have

$$
\left(c-\sigma_{c}\right) \theta \mathfrak{P}=1 \text { in } C l(D) .
$$

Let $w$ be a primitive root $\bmod p$. Then $D=\langle 2\rangle=\left\langle w^{g}\right\rangle \subseteq G_{0}=(\mathbb{Z} / p \mathbb{Z})^{\times}$. It follows that we can assume

$$
\sigma_{w^{t g+s}}(x)=x_{s+1}, \quad t \in \mathbb{Z}, s=0,1, \ldots, g-1 .
$$

Let $k_{c, a}=\left[\frac{c a}{p}\right]$ for any integer $a$. We have

$$
\begin{aligned}
\left(c-\sigma_{c}\right) \theta & =\left(c-\sigma_{c}\right) \sum_{a \in(\mathbb{Z} / p \mathbb{Z})^{\times}}\left\{\frac{a}{p}\right\} \sigma_{a}^{-1} \\
& =\sum_{a}\left(c\left\{\frac{a}{p}\right\}-\left\{\frac{c a}{p}\right\}\right) \sigma_{a}^{-1} \\
& =\sum_{a=1}^{p-1} k_{c, a} \sigma_{a}^{-1} \\
& =\sum_{s=0}^{p-2} k_{c, w^{-s}} \sigma_{w}^{s} \\
& =\sum_{t=0}^{f-1} \sum_{s=0}^{g-1} k_{c, w^{-(t g+s)}} \sigma_{w}^{t g+s}
\end{aligned}
$$


Then by (5.11) we have

$$
\begin{aligned}
0 & =\mathfrak{P}^{\sum_{t=0}^{f-1} \sum_{s=0}^{g-1} k_{c, w}-(t g+s)} \sigma_{w}^{t g+s} \\
& =\sum_{t=0}^{f-1} \sum_{s=0}^{g-1} k_{c, w^{-(t g+s)}} \sigma_{w}^{t g+s}(x) \\
& =\sum_{t=0}^{f-1} \sum_{s=0}^{g-1} k_{c, w^{-(t g+s)}} x_{s+1} \\
& =\sum_{s=1}^{g} m_{c, s} \sum_{t=0}^{f-1} k_{c, w^{-t g+s-1}} x_{s} .
\end{aligned}
$$

If we set

$$
m_{c, s}=\sum_{t=0}^{f-1} k_{c, w^{-t g+s-1}}
$$

we have $p-1$ linear equations

$$
\sum_{s=1}^{g} m_{c, s} x_{s}=0, \quad c=1,2, \ldots p-1 .
$$

We now combine these $p-1$ equations, together with the equation (5.7) and the $u$ equations (5.10), to give a whole collection of equations

$$
X M^{T}=0
$$

where $M$ is a $(p+u) \times g$ matrix with integer entries made of the coefficients of all the $p+u$ equations and $X=\left(x_{1}, x_{2}, \ldots, x_{g}\right)$. To simplify these relations of $x_{1}, x_{2}, \ldots, x_{g}$, we need to calculate the Hermite normal form of $M$. By the well-known result (c.f. [1, § 2.4.2]) for the existence of the Hermite normal form, there exists a unique matrix $U \in \mathrm{GL}_{p+u}(\mathbb{Z})$, such that $H=M^{T} U$ is a Hermite normal form. It follows from (5.14) that

$$
X H=0 .
$$

In fact, $H$ can be obtained by applying a finite sequence of elementary row operations over $\mathbb{Z}$ from $M^{T}$.

Now with the help of a computer and using a simple program or a computer algebra system, we can calculate the individual Hermite normal form $H$ for $p=31$ and 151 ( $u=3$ and 5 , respectively). Thus we obtain the relation

$$
\left(x_{1}, x_{2}, x_{3}\right)\left(\begin{array}{ccc}
18 & 14 & 3 \\
0 & 2 & 1 \\
0 & 0 & 1
\end{array}\right)=0
$$

and

$$
\left(x_{1}, x_{2}, \ldots, x_{5}\right)\left(\begin{array}{ccccc}
3934 & 1430 & 390 & 464 & 2457 \\
0 & 2 & 0 & 0 & 1 \\
0 & 0 & 2 & 0 & 1 \\
0 & 0 & 0 & 2 & 1 \\
0 & 0 & 0 & 0 & 1
\end{array}\right)=0
$$


where we omit $x_{u+1}, \ldots, x_{g}$ and other parts of $H$ since $x_{u+k}=-x_{k}$.

Using these computational results, we can turn to

Proof of the Theorem 1.5. If $p=31$ the first column of the matrix in (5.15) tells us that $18 x_{1}=0$ in $C l(D)$. By [17, Table §3] we know $h_{31}^{-}$is odd and hence $h_{31}=h\left(K_{0}\right)=h_{31}^{-} h_{31}^{+}=h_{31}^{-}$is also odd. It follows that $9 x_{1}=0$ and $\operatorname{ord}\left(x_{1}\right)=1,3$ or 9 .

We claim that $\operatorname{ord}\left(x_{1}\right)=9$. Recall $F=\mathbb{Q}(\sqrt{-p})=\mathbb{Q}(\sqrt{-31}) \subseteq D$ and let $\mathfrak{p}_{F}=\mathfrak{P}_{1} \cap \mathfrak{o}_{F}$. It is easy to know that $h(F)=3$ and $\mathfrak{p}_{F}$ has order 3 in $C l(F)$. If $\operatorname{ord}\left(x_{1}\right)=1$, i.e. $\mathfrak{P}_{1}=1$ in $C l(D)$, then taking norm gives $\mathfrak{p}_{F}=1$ in $C l(F)$, which is a contradiction. If $\operatorname{ord}\left(x_{1}\right)=3$, then $\left\langle x_{1}\right\rangle \cong \mathbb{Z} / 3 \mathbb{Z}$ and we may assume $x_{1}=1 \bmod 3$. Then the second column of the matrix reads $14 x_{1}+2 x_{2}=0$, or $x_{2}=-7 x_{1}$. Hence $x_{2}=2 x_{1}=-1 \bmod 3$ and similarly $x_{3}=1 \bmod 3$. Thus $x_{k}= \pm 1 \bmod 3 \in \mathbb{Z} / 3 \mathbb{Z}$ for all $k=1,2, \ldots, 6$. But we know three of all six $x_{k}$ 's (i.e. $\mathfrak{P}_{k}$ 's) lie over $\mathfrak{p}_{F}$. Suppose that $\mathfrak{p}_{F} \mathfrak{o}_{D}=\mathfrak{P}_{k_{1}} \mathfrak{P}_{k_{2}} \mathfrak{P}_{k_{3}}$. If all these three $x_{k_{1}}, x_{k_{2}}, x_{k_{3}}$ are the same, say 1 (mod 3$)$, then since $C l(F) \longrightarrow C l(D)$ is injective (Proposition 2.2) we have $\mathfrak{p}_{F}=1$ in $C l(F)$, a contradiction. Otherwise we may assume $x_{k_{1}}=-x_{k_{2}}=1$ and then $x_{k_{1}}+x_{k_{2}}=0$. Taking norm gives $\mathfrak{p}_{F}^{2}=1$, which is also false.

Thus we have ord $\left(x_{1}\right)=9$ and using the matrix again we obtain

$$
\left(x_{1}, x_{2}, \ldots, x_{6}\right)=(1,2,4,-1,-2,-4)
$$

are all in $\left\langle x_{1}\right\rangle \cong \mathbb{Z} / 9 \mathbb{Z}$. We now apply Proposition 5.5. Let $n=1,3 \ldots$ and solve the equation (5.3) modulo 9. A simple calculation tells us that $n_{0}=3$ in the proposition and all the solutions $\left(n_{1}, n_{2}, \ldots, n_{6}\right)$ to (5.3) corresponding to $n=n_{0}$ are

$$
\begin{aligned}
& (2,0,1,1,3,2),(2,2,0,1,1,3),(3,0,3,0,3,0),(3,2,2,0,1,1) \text { and } \\
& (1,3,2,2,0,1),(1,1,3,2,2,0),(0,3,0,3,0,3),(0,1,1,3,2,2) .
\end{aligned}
$$

Obviously the corresponding $Z_{p}$ 's are nonempty and pairwise different. Hence we obtain by the proposition the non-existence of GBFs of type $\left[n \leq 3,2 \times 31^{e}\right]$.

The argument for $p=151$ is similar. Using the matrix in (5.16) we know that $2 \times 7 \times 281 x_{1}=0$. Noting that $h_{151}$ is also odd, we find that $\operatorname{ord}\left(x_{1}\right)=7,281$ or 1967 . In this case $F=\mathbb{Q}(\sqrt{-157})$. Knowing that $h(F)=7$ and $\mathfrak{p}_{F}$ has order 7 in $C l(F)$, the candidate order 1 and 7 can be removed by the previous method. If we have $281 x_{1}=0$, taking norm gives $\mathfrak{p}_{F}^{281}=1$, which contradicts to $\operatorname{ord}\left(\mathfrak{p}_{F}\right)=7$. Thus $\operatorname{ord}\left(x_{1}\right)=1967$ and we obtain $x_{1}, \ldots, x_{10} \in\left\langle x_{1}\right\rangle \cong \mathbb{Z} / 1967 \mathbb{Z}$ and

$$
\begin{gathered}
\left(x_{1}, x_{2}, \ldots, x_{5}\right)=(1,-715,-195,-232,335) \\
x_{5+k}=-x_{k}, k=1,2, \ldots, 5 .
\end{gathered}
$$

Let $n=1,3 \ldots$ and solve the equation (5.3) modulo 1967. We find that $n_{0}=5$ in Proposition 5.5 and all the solutions $\left(n_{1}, n_{2}, \ldots, n_{6}\right)$ to (5.3) corresponding to $n=n_{0}$ are

$$
\begin{aligned}
& (4,1,4,1,5,1,4,1,4,0)(5,3,2,5,5,0,2,3,0,0) \text { and } \\
& (1,4,1,4,0,4,1,4,1,5)(0,2,3,0,0,5,3,2,5,5),
\end{aligned}
$$

whose corresponding $Z_{p}$ 's are nonempty and pairwise different. Again the proposition implies the non-existence of GBFs of type $\left[n \leq 5,2 \times 151^{e}\right]$. The proof is done.

\section{Non-eXistence Results by PARI/GP AND other Remarks}

If $p \equiv 1(\bmod 8)$ and $\operatorname{ord}_{p^{e}}(2)$ is odd, there is no proof for non-existence of the type $\left[n, 2 p^{e}\right]$. The Stickelberger relation method used in the previous section is not available since $K$ dos not contain a imaginary quadratic field and most $h_{p}^{+}$'s equal to 1 (Miller's conjectures). 
However, if the degree of the decomposition field of 2 is small, we could use PARI to calculate the relations of $\mathfrak{P}_{k}$ 's in $C l(D)$ (c.f. Section 5 , especially (5.1)). For example, we obtain without proof that

Conjecture 6.1. Let $e$ and $n$ be positive integers and $n$ be odd. Then there is no GBFs of type $\left[1 \leq n \leq 7,2 \times 233^{e}\right]$.

The method combining Stickelberger relations and Hermite normal form is not a systematic one, so we hope that we could develop this method to obtain non-existence results for a class of types.

Acknowledgment The author would like to thank Yingpu Deng and Yupeng Jiang for many helpful discussions and comments.

\section{REFERENCES}

[1] H. Cohen, A course in computational algebraic number theory, Graduate Texts in Mathematics, vol. 138, Springer, New York, 1993.

[2] John Francis Dillon, Elementary hadamard difference sets, Ph.D. thesis, University of Maryland Ph. D. dissertation, 1974.

[3] Ke Qin Feng and Feng Mei Liu, Non-existence of some generalized bent functions, Acta Mathematica Sinica 19 (2003), no. 1, 39-50.

[4] Keqin Feng, Generalized bent functions and class group of imaginary quadratic fields, Science in China Series A: Mathematics 44 (2001), no. 5, 562-570.

[5] Keqin Feng and Fengmei Liu, New results on the nonexistence of generalized bent functions, Information Theory, IEEE Transactions on 49 (2003), no. 11, 3066-3071.

[6] Ralph Greenberg, Topics in iwasawa theory, http://http://www.math.washington.edu/ greenber/chap1.pdf 2006.

[7] Masatoshi Ikeda, A remark on the non-existence of generalized bent functions, Number Theory and Its Applications (Ankara, 1996) (1998), 109-119.

[8] Yupeng Jiang and Yingpu Deng, New results on nonexistence of generalized bent functions, Designs, Codes and Cryptography 75 (2015), no. 3, 375-385.

[9] P Vijay Kumar, Robert A. Scholtz, and Lloyd R. Welch, Generalized bent functions and their properties, Journal of Combinatorial Theory, Series A 40 (1985), no. 1, 90-107.

[10] Jianing Li and Yingpu Deng, Nonexistence of two classes of generalized bent functions, arXiv preprint arXiv:1507.06886 (2015).

[11] H. Liu, K. Feng, and R. Feng, Nonexistence of generalized bent functions from $\mathbb{Z}_{2}^{n}$ to $\mathbb{Z}_{m}$, preprint (2015).

[12] Daniel A Marcus, Number fields, Springer, 1977.

[13] John Miller, Real cyclotomic fields of prime conductor and their class numbers, Mathematics of Computation 84 (2015), no. 295, 2459-2469.

[14] John D Olsen, Robert Scholtz, Lloyd R Welch, et al., Bent-function sequences, Information Theory, IEEE Transactions on $\mathbf{2 8}$ (1982), no. 6, 858-864.

[15] DY Pei, On non-existence of generalized bent functions, LN in pure and applied math 141 (1993), 165-172.

[16] Oscar S Rothaus, On "bent" functions, Journal of Combinatorial Theory, Series A 20 (1976), no. 3, $300-305$.

[17] Lawrence C Washington, Introduction to cyclotomic fields, 2nd ed., Graduate Texts in Mathematics, vol. 83, Springer, New York, 1997.

[18] PARI/GP, http://pari.math.u-bordeaux.fr/

SKLOIS, Institute of Information Engineering, CAS, Beijing 100093, China

E-mail address: lvchang@amss.ac.cn

Key Laboratory of Mathematics Mechanization, NCMis, Academy of Mathematics and Systems SciENCE, CAS, BeiJing 100190, China

E-mail address: lijianing19891026@163.com

\footnotetext{
${ }^{1}$ See [18, a widely used computer algebra system designed for fast computation in number theory and originally developed by Henri Cohen and his co-workers
} 